\title{
DDMVAC with Growth Factor
}

National Cancer Institute

\section{Source}

National Cancer Institute. DDMVAC with Growth Factor. NCI Thesaurus. Code C159436.

A dose-dense chemotherapy regimen consisting of methotrexate, vinblastine,

doxorubicin, cisplatin (DDMVAC) and the addition of a selected growth factor to support the (neo)adjuvant treatment of bladder cancer. 